\title{
Source Plasma
}

National Cancer Institute

\section{Source}

National Cancer Institute. Source Plasma. NCI Thesaurus. Code C133347.

Plasma derived from single units of whole blood, plasma, or as a by-product in the preparation of blood components from whole blood, for use in the manufacturing of licensed or unlicensed products. 